\title{
On the Oscillation for Second-Order Half-Linear Neutral Delay Dynamic Equations on Time Scales
}

\author{
Quanxin Zhang and Xia Song \\ Department of Mathematics, Binzhou University, Shandong 256603, China \\ Correspondence should be addressed to Quanxin Zhang; 3314744@163.com \\ Received 14 January 2014; Accepted 26 February 2014; Published 28 April 2014 \\ Academic Editor: Shurong Sun
}

Copyright ( 2014 Q. Zhang and X. Song. This is an open access article distributed under the Creative Commons Attribution License, which permits unrestricted use, distribution, and reproduction in any medium, provided the original work is properly cited.

We discuss oscillation criteria for second-order half-linear neutral delay dynamic equations on time scales by using the generalized Riccati transformation and the inequality technique. Under certain conditions, we establish four new oscillation criteria. Our results in this paper are new even for the cases of $\mathbb{T}=\mathbb{R}$ and $\mathbb{T}=\mathbb{Z}$.

\section{Introduction}

In recent years, the research results relevant to oscillation of second-order dynamic equations on time scales are emerging, such as [1-7]. The research results of oscillation for the second-order linear, nonlinear, or half-linear dynamic equations can be found in [8-23]. On the basis of the above work, we will study the oscillatory behavior of all solutions of second-order half-linear neutral delay dynamic equation in this paper, which is given as follows:

$$
\begin{array}{r}
\left(a(t) \Phi\left(z^{\Delta}(t)\right)\right)^{\Delta}+q(t) f(\Phi(x(\tau(t))))=0, \\
t \in \mathbb{T}, \quad t \geq t_{0},
\end{array}
$$

where $\Phi(s)=|s|^{\gamma-2} s, z(t)=x(t)+r(t) x(\tau(t)), \gamma>1$. In this paper, we give the following hypotheses.

$\left(\mathrm{H}_{1}\right) \mathbb{T}$ is a time scale (i.e., a nonempty closed subset of the real numbers $\mathbb{R}$ ) which is unbounded above and for $t_{0} \in \mathbb{T}$ with $t_{0}>0$; we define the time scale interval of the form $\left[t_{0}, \infty\right)_{\mathbb{T}}$ by $\left[t_{0}, \infty\right)_{\mathbb{T}}=\left[t_{0}, \infty\right) \cap \mathbb{T}$.

$\left(\mathrm{H}_{2}\right) a, r, q: \mathbb{T} \rightarrow \mathbb{R}$ are positive rd-continuous functions such that $0<r(t)<1$.

$\left(\mathrm{H}_{3}\right) \tau: \mathbb{T} \rightarrow \mathbb{T}$ is a strictly increasing and differentiable function such that

$$
\tau(t) \leq t, \quad \lim _{t \rightarrow \infty} \tau(t)=\infty, \tau(\mathbb{T})=\mathbb{T} .
$$

$\left(\mathrm{H}_{4}\right) f: \mathbb{R} \rightarrow \mathbb{R}$ is a continuous function such that, for some positive constant $L$,

$$
\frac{f(x)}{x} \geq L \quad \forall x \neq 0 .
$$

By a solution of (1), we mean a nontrivial real-valued function $x$ satisfying (1) for $t \in \mathbb{T}$. A solution $x$ of (1) is called oscillatory if it is neither eventually positive nor negative; otherwise, it is called nonoscillatory. Equation (1) is called oscillatory if all its solutions are oscillatory. Our attention is restricted on those solutions of (1) which are not eventually identically zero.

The purpose of this paper is to establish the oscillation criteria of Philos [24] for (1). When

$$
\int_{t_{0}}^{\infty}\left[\frac{1}{a(t)}\right]^{1 /(\gamma-1)} \Delta t=\infty,
$$

the two famous results of Philos [24] about oscillation of second-order linear differential equations are extended to (1) in this paper. At the same time, when

$$
\int_{t_{0}}^{\infty}\left[\frac{1}{a(t)}\right]^{1 /(\gamma-1)} \Delta t<\infty,
$$

we obtain two criteria of (1) about that each solution is either oscillatory or converges to zero. 
The paper is organized as follows. In Section 2, we present some basic definitions and useful results about the theory of calculus on time scales. In Section 3, we give six lemmas. Section 4 introduces the main results of this paper. We establish four new oscillatory criteria when the condition (4) or (5) holds, respectively, for the solutions of (1).

\section{Some Preliminaries}

On the time scale $\mathbb{}$ we define the forward and backward jump operators by

$$
\sigma(t)=\inf \{s \in \mathbb{T}: s>t\}, \quad \rho(t)=\sup \{s \in \mathbb{T}: s<t\} .
$$

A point $t \in \mathbb{T}$ is said to be left-dense if it satisfies $\rho(t)=t$, right-dense if it satisfies $\sigma(t)=t$, left-scattered if it satisfies $\rho(t)<t$, and right-scattered if it satisfies $\sigma(t)>t$. The graininess $\mu$ of the time scale is defined by $\mu(t)=\sigma(t)-t$. For a function $f: \mathbb{T} \rightarrow \mathbb{R}$, the (delta) derivative is defined by

$$
f^{\Delta}(t)=\frac{f(\sigma(t))-f(t)}{\sigma(t)-t}
$$

if $f$ is continuous at $t$ and $t$ is right-scattered. If $t$ is rightdense, then the derivative is defined by

$$
f^{\Delta}(t)=\lim _{s \rightarrow t^{+}} \frac{f(t)-f(s)}{t-s},
$$

provided this limit exists. A function $f: \mathbb{T} \rightarrow \mathbb{R}$ is said to be rd-continuous if it is continuous at each right-dense point and if there exists a finite left limit at all left-dense points. Denoted by $C_{\text {rd }}(\mathbb{T}, \mathbb{R})$ the set of rd-continuous functions on $\mathbb{V}$ and $C_{\text {rd }}^{1}(\mathbb{T}, \mathbb{R})$ the set of differentiable function on $\mathbb{T}$, whose derivative is rd-continuous. The derivative $f^{\Delta}$ of $f$, the shift $f^{\sigma}$ of $f$, and the graininess $\mu$ are related by the formula

$$
f^{\sigma}=f+\mu f^{\Delta} \quad \text { where } f^{\sigma}=f \circ \sigma .
$$

We will make use of the following product and quotient rules for the derivative of the product $f g$ and the quotient $f / g$ of two differentiable functions $f$ and $g$ :

$$
\begin{aligned}
(f g)^{\Delta}(t) & =f^{\Delta}(t) g(t)+f(\sigma(t)) g^{\Delta}(t) \\
& =f(t) g^{\Delta}(t)+f^{\Delta}(t) g(\sigma(t)), \\
\left(\frac{f}{g}\right)^{\Delta}(t) & =\frac{f^{\Delta}(t) g(t)-f(t) g^{\Delta}(t)}{g(t) g(\sigma(t))} \text { if } g g^{\sigma} \neq 0 .
\end{aligned}
$$

For $b, c \in \mathbb{T}$, the Cauchy integral of $f^{\Delta}$ is defined by

$$
\int_{b}^{c} f^{\Delta}(t) \Delta t=f(c)-f(b) .
$$

The integration by parts formula reads

$$
\begin{aligned}
& \int_{b}^{c} f^{\Delta}(t) g(t) \Delta t \\
& \quad=f(c) g(c)-f(b) g(b)-\int_{b}^{c} f^{\sigma}(t) g^{\Delta}(t) \Delta t,
\end{aligned}
$$

and the infinite integral is defined by

$$
\int_{b}^{\infty} f(s) \Delta s=\lim _{t \rightarrow \infty} \int_{b}^{t} f(s) \Delta s .
$$

For more details, see $[8,9]$.

\section{Several Lemmas}

In this section, we present five lemmas that will be needed in the proofs of our results in Section 4. Lemma 1 is the theorem 1.93 of [8]; Lemma 2 is the simple corollary of theorem 1.90 in [8]; Lemma 3 is the theorem 41 in [25]; and Lemma 4 is the theorem 3 in [26].

Lemma 1. Assume that $v: \mathbb{T} \rightarrow \mathbb{R}$ is strictly increasing and $\widetilde{\mathbb{T}}:=v(\mathbb{T})$ is a time scale. Let $w: \widetilde{\mathbb{T}} \rightarrow \mathbb{R}$. If $v^{\Delta}(t)$ and $w^{\widetilde{\Delta}}(v(t))$ exist on $\mathbb{T}^{k}$, where

$$
\mathbb{T}^{k}= \begin{cases}\mathbb{T} \backslash(\rho(\sup \mathbb{T}), \sup \mathbb{\mathbb { T }}], & \text { if } \sup \mathbb{T}<\infty, \\ \mathbb{T}, & \text { if } \sup \mathbb{T}=\infty,\end{cases}
$$

then

$$
(w \circ v)^{\Delta}=\left(w^{\tilde{\Delta}} \circ v\right) v^{\Delta} .
$$

Lemma 2. If $x$ is differentiable, then

$$
\left(x^{\gamma}\right)^{\Delta}=\gamma x^{\Delta} \int_{0}^{1}\left[h x^{\sigma}+(1-h) x\right]^{\gamma-1} \mathrm{~d} h .
$$

Lemma 3. Assume that $X$ and $Y$ are nonnegative real numbers, then

$$
\lambda X Y^{\lambda-1}-X^{\lambda} \leq(\lambda-1) Y^{\lambda} \quad \forall \lambda>1,
$$

where the equality holds if and only if $X=Y$.

Lemma 4. Let $a, b \in \mathbb{T}$ with $a<b$. Then for positive $r d$-continuous functions $f, g:[a, b] \rightarrow \mathbb{R}$ we have

$$
\int_{a}^{b}|f(s) g(s)| \Delta s \leq\left(\int_{a}^{b}|f(s)|^{p} \Delta s\right)^{1 / p}\left(\int_{a}^{b}|g(s)|^{q} \Delta s\right)^{1 / q},
$$

where $p>1$ and $1 / p+1 / q=1$

Lemma 5. Assume that $\left(H_{1}\right)-\left(H_{4}\right)$ and (4) hold. Let $x(t)$ be an eventually positive solution of (1). Then there exists $t_{1} \epsilon$ $\left[t_{0}, \infty\right)_{\mathbb{T}}$ such that

$$
z^{\Delta}(t)>0, \quad\left(a(t)\left|z^{\Delta}(t)\right|^{\gamma-2} z^{\Delta}(t)\right)^{\Delta}<0
$$

Proof. Suppose that $x(t)$ is an eventually positive solution of (1). There exists $t_{1} \in\left[t_{0}, \infty\right)_{\mathbb{V}}$ such that $x(t)>0$ and $x(\tau(t))>$ 0 for $t \in\left[t_{1}, \infty\right)_{\mathbb{T}}$. From the definition of $z(t)$, we get $z(t)>0$ for $t \in\left[t_{1}, \infty\right)_{\mathbb{T}}$, and at the same time for $t \in\left[t_{1}, \infty\right)_{\mathbb{T}}$, from (1) we get

$$
\left(a(t)\left|z^{\Delta}(t)\right|^{\gamma-2} z^{\Delta}(t)\right)^{\Delta}<0
$$


Hence, $a(t)\left|z^{\Delta}(t)\right|^{\gamma-2} z^{\Delta}(t)$ is decreasing. So, $z^{\Delta}(t)$ is either eventually positive or eventually negative. Therefore, for arbitrary $t \in\left[t_{1}, \infty\right)_{\mathbb{T}}$, we have

$$
z^{\Delta}(t)>0
$$

Otherwise, we assume that (22) is not satisfied, then there exits $t_{2} \in\left[t_{1}, \infty\right)_{\mathbb{T}}$ such that $z^{\Delta}(t)<0$ for all $t \in\left[t_{2}, \infty\right)_{\mathbb{T}}$. By (21) we have

$$
a(t)\left|z^{\Delta}(t)\right|^{\gamma-2} z^{\Delta}(t) \leq a\left(t_{2}\right)\left|z^{\Delta}\left(t_{2}\right)\right|^{\gamma-2} z^{\Delta}\left(t_{2}\right)=-M^{\gamma-1}
$$

for $t \in\left[t_{2}, \infty\right)_{\mathbb{T}}$, where $M=\left[a\left(t_{2}\right)\right]^{1 /(\gamma-1)}\left|z^{\Delta}\left(t_{2}\right)\right|>0$. By (23), we get

$$
\left(-z^{\Delta}(t)\right)^{\gamma-1} \geq \frac{M^{\gamma-1}}{a(t)}, \quad t \in\left[t_{2}, \infty\right)_{\mathbb{T}}
$$

that is

$$
z^{\Delta}(t) \leq-M\left[\frac{1}{a(t)}\right]^{1 /(\gamma-1)}, \quad t \in\left[t_{2}, \infty\right)_{\mathbb{T}} .
$$

After integrating the two sides of inequality (25) from $t_{2}$ to $t \in\left[t_{2}, \infty\right)_{\mathbb{V}}$, we have

$$
z(t) \leq z\left(t_{2}\right)-M \int_{t_{2}}^{t}\left[\frac{1}{a(s)}\right]^{1 /(\gamma-1)} \Delta s, \quad t \in\left[t_{2}, \infty\right)_{\mathbb{T}} .
$$

Nextly, we find the limits of the two sides of (26) when $t \rightarrow$ $\infty$. From (4), we get $\lim _{t \rightarrow \infty} z(t)=-\infty$. Therefore, $z(t)$ is eventually negative, which is contradictory to $z(t)>0$. So the inequality (22) holds. This completes the proof.

\section{Main Results}

Firstly, the two famous results of Philos [24] about oscillation of second-order linear differential equations are extended to (1) when condition (4) is satisfied.

Theorem 6. Assume that $\left(H_{1}\right)-\left(H_{4}\right)$ and (4) hold. Let $H$ : $D_{\mathbb{T}} \equiv\left\{(t, s): t \geq s \geq t_{0}, t, s \in\left[t_{0}, \infty\right)_{\mathbb{T}}\right\} \rightarrow \mathbb{R}$ be rdcontinuous function, such that

$$
\begin{gathered}
H(t, t)=0, \quad t \geq t_{0} ; \quad H(t, s)>0, \quad t>s \geq t_{0}, \\
t, s \in\left[t_{0}, \infty\right)_{\mathbb{T}}
\end{gathered}
$$

and $H$ has a nonpositive continuous $\Delta$-partial derivative $H^{\Delta_{s}}(t, s)$ with respect to the second variable and satisfies

$$
\begin{aligned}
& 0<\inf _{s \geq T_{0}}\left[\liminf _{t \rightarrow \infty} \frac{H(t, s)}{H\left(t, T_{0}\right)}\right] \leq \infty, \quad T_{0} \in\left[t_{0}, \infty\right)_{\mathbb{T}}, \\
& -H^{\Delta_{s}}(t, s)=h(t, s)(H(t, s))^{(\gamma-1) / \gamma}, \quad(t, s) \in D_{\mathbb{T}},
\end{aligned}
$$

where $h: D_{\mathbb{T}} \rightarrow \mathbb{R}$ is a rd-continuous function. If there exist a positive and differentiable function $\delta: \mathbb{T} \rightarrow \mathbb{R}$ such that
$\delta^{\Delta}(t) \geq 0$ for $t \in\left[t_{0}, \infty\right)_{\mathbb{T}}$, and a real $r d$-continuous function $\Psi:\left[t_{0}, \infty\right)_{\mathbb{V}} \rightarrow \mathbb{R}$ such that

$$
\begin{gathered}
\limsup _{t \rightarrow \infty} \frac{1}{H\left(t, T_{0}\right)} \int_{T_{0}}^{t} \frac{a(\tau(s))}{\left(\delta(s) \tau^{\Delta}(s)\right)^{\gamma-1}} G_{+}^{\gamma}(t, s) \Delta s<\infty, \\
\int_{T_{0}}^{\infty} \frac{\delta(s) \tau^{\Delta}(s)}{(a(\tau(s)))^{1 /(\gamma-1)}}\left(\frac{\Psi_{+}(\sigma(s))}{\delta(\sigma(s))}\right)^{\gamma /(\gamma-1)} \Delta s=\infty, \\
\limsup _{t \rightarrow \infty} \frac{1}{H(t, T)} \\
\times \int_{T}^{t}\left[L H(t, s) \delta(s) q(s)(1-r(\tau(s)))^{\gamma-1}\right. \\
\left.-\frac{a(\tau(s))}{\gamma^{\gamma}\left(\delta(s) \tau^{\Delta}(s)\right)^{\gamma-1}} G_{+}^{\gamma}(t, s)\right] \Delta s
\end{gathered}
$$

$$
\geq \Psi(T),
$$

where $G(t, s)=\delta^{\Delta}(s)(H(t, s))^{1 / \gamma}-\delta(s) h(t, s), G_{+}(t, s)=$ $\max \{0, G(t, s)\}, \Psi_{+}(t)=\max \{0, \Psi(t)\}, T \in\left[T_{0}, \infty\right)_{\mathbb{T}}$, then (1) is oscillatory on $\left[t_{0}, \infty\right)_{\mathbb{T}}$.

Proof. Assume that (1) has a nonoscillatory solution $x(t)$ on $\left[t_{0}, \infty\right)_{\mathbb{T}}$. Without loss of generality we may assume that there exists a $t_{1} \in\left[t_{0}, \infty\right)_{\mathbb{T}}$, such that $x(t)>0$ and $x[\tau(t)]>0$ for all $t \in\left[t_{1}, \infty\right)_{\mathbb{T}}$. By the definition of $z(t)$, it follows that

$$
\begin{aligned}
x(t) & =z(t)-r(t) x(\tau(t)) \\
& \geq z(t)-r(t) z(\tau(t)) \\
& \geq(1-r(t)) z(t), \quad t \in\left[t_{1}, \infty\right)_{\mathbb{T}} .
\end{aligned}
$$

Since $\lim _{t \rightarrow \infty} \tau(t)=\infty$, there exists $T_{0} \in\left[t_{0}, \infty\right)_{\mathbb{T}}$, such that $\tau(t) \geq t_{1}$, for all $t \in\left[T_{0}, \infty\right)_{\mathbb{T}}$. Then for $t \in\left[T_{0}, \infty\right)_{\mathbb{T}}$, we have

$$
x(\tau(t)) \geq(1-r(\tau(t))) z(\tau(t)) .
$$

By Lemma 5 and $\left(\mathrm{H}_{3}\right)$, we obtain that

$$
\frac{1}{z \circ \tau} \geq \frac{1}{z \circ \tau^{\sigma}}, \quad a\left(z^{\Delta}\right)^{\gamma-1} \geq a^{\sigma}\left(z^{\Delta \sigma}\right)^{\gamma-1}
$$

on $\left[T_{0}, \infty\right)_{\mathbb{T}}\left(\right.$ where $\left(z^{\Delta}\right)^{\sigma}$ is short hand for $\left.z^{\Delta \sigma}\right)$, and

$$
z^{\Delta} \circ \tau \geq \frac{\left(a^{\sigma}\right)^{1 /(\gamma-1)}}{(a \circ \tau)^{1 /(\gamma-1)}} z^{\Delta \sigma}
$$

holds. Moreover, using Lemmas 2 and 5, it follows that

$$
\begin{aligned}
& {\left[(z \circ \tau)^{\gamma-1}\right]^{\Delta}} \\
& \quad=(\gamma-1)(z \circ \tau)^{\Delta} \int_{0}^{1}\left[h\left(z \circ \tau^{\sigma}\right)+(1-h)(z \circ \tau)\right]^{\gamma-2} \mathrm{~d} h \\
& \quad \geq(\gamma-1)(z \circ \tau)^{\Delta} \int_{0}^{1}[h(z \circ \tau)+(1-h)(z \circ \tau)]^{\gamma-2} \mathrm{~d} h \\
& \quad=(\gamma-1)(z \circ \tau)^{\gamma-2}(z \circ \tau)^{\Delta} .
\end{aligned}
$$


In Lemma 1 , let $v=\tau, w=z$, and $\mathbb{T}$ is unbounded above by $\left(\mathrm{H}_{1}\right)$, so $\mathbb{\mathbb { T }}^{k}=\mathbb{T}$, and $\widetilde{\mathbb{T}}=v(\mathbb{\mathbb { T }})=\tau(\mathbb{\mathbb { T }})=\mathbb{\mathbb { V }}$ by $\left(\mathrm{H}_{3}\right)$; using Lemma 1, we get

$$
(z \circ \tau)^{\Delta}=\left(z^{\Delta} \circ \tau\right) \tau^{\Delta}
$$

Thus

$$
\left[(z \circ \tau)^{\gamma-1}\right]^{\Delta} \geq(\gamma-1)(z \circ \tau)^{\gamma-2}\left(z^{\Delta} \circ \tau\right) \tau^{\Delta} .
$$

By the above inequality and the first inequality in (35), we obtain that

$$
\frac{\left[(z \circ \tau)^{\gamma-1}\right]^{\Delta}}{(z \circ \tau)^{\gamma-1}} \geq \frac{(\gamma-1)\left(z^{\Delta} \circ \tau\right) \tau^{\Delta}}{z \circ \tau^{\sigma}}
$$

holds on $\left[T_{0}, \infty\right)_{\mathbb{T}}$. Now we define the function $W$ by

$$
W=\delta \frac{a\left(z^{\Delta}\right)^{\gamma-1}}{(z \circ \tau)^{\gamma-1}}
$$

Then we have $W>0$ on $\left[T_{0}, \infty\right)_{\mathbb{T}}$, and

$$
\begin{aligned}
& W^{\Delta} \stackrel{(10)}{=} \frac{\delta}{(z \circ \tau)^{\gamma-1}}\left[a\left(z^{\Delta}\right)^{\gamma-1}\right]^{\Delta} \\
& +a^{\sigma}\left(z^{\Delta \sigma}\right)^{\gamma-1} \frac{(z \circ \tau)^{\gamma-1} \delta^{\Delta}-\delta\left[(z \circ \tau)^{\gamma-1}\right]^{\Delta}}{(z \circ \tau)^{\gamma-1}\left(z \circ \tau^{\sigma}\right)^{\gamma-1}} \\
& \stackrel{(1)\left(\mathrm{H}_{4}\right)}{\leq}-\frac{L q \delta(x \circ \tau)^{\gamma-1}}{(z \circ \tau)^{\gamma-1}} \\
& +a^{\sigma}\left(z^{\Delta \sigma}\right)^{\gamma-1} \frac{(z \circ \tau)^{\gamma-1} \delta^{\Delta}-\delta\left[(z \circ \tau)^{\gamma-1}\right]^{\Delta}}{(z \circ \tau)^{\gamma-1}\left(z \circ \tau^{\sigma}\right)^{\gamma-1}} \\
& \stackrel{(34)}{\leq}-L q \delta(1-r \circ \tau)^{\gamma-1} \\
& +a^{\sigma}\left(z^{\Delta \sigma}\right)^{\gamma-1} \frac{(z \circ \tau)^{\gamma-1} \delta^{\Delta}-\delta\left[(z \circ \tau)^{\gamma-1}\right]^{\Delta}}{(z \circ \tau)^{\gamma-1}\left(z \circ \tau^{\sigma}\right)^{\gamma-1}} \\
& \stackrel{(41)}{=}-L q \delta(1-r \circ \tau)^{\gamma-1}+\frac{\delta^{\Delta}}{\delta^{\sigma}} W^{\sigma} \\
& -\frac{\delta a^{\sigma}\left(z^{\Delta \sigma}\right)^{\gamma-1}\left[(z \circ \tau)^{\gamma-1}\right]^{\Delta}}{(z \circ \tau)^{\gamma-1}\left(z \circ \tau^{\sigma}\right)^{\gamma-1}} \\
& \stackrel{(40)}{\leq}-L q \delta(1-r \circ \tau)^{\gamma-1} \\
& +\delta^{\Delta} \frac{W^{\sigma}}{\delta^{\sigma}}-\frac{(\gamma-1) \delta a^{\sigma}\left(z^{\Delta \sigma}\right)^{\gamma-1}\left(z^{\Delta} \circ \tau\right) \tau^{\Delta}}{\left(z \circ \tau^{\sigma}\right)^{\gamma}} \\
& \stackrel{(36)}{\leq}-L q \delta(1-r \circ \tau)^{\gamma-1}+\delta^{\Delta} \frac{W^{\sigma}}{\delta^{\sigma}} \\
& -\frac{(\gamma-1) \delta \tau^{\Delta}\left(a^{\sigma}\right)^{\gamma /(\gamma-1)}\left(z^{\Delta \sigma}\right)^{\gamma}}{(a \circ \tau)^{1 /(\gamma-1)}\left(z \circ \tau^{\sigma}\right)^{\gamma}}
\end{aligned}
$$

$$
\begin{gathered}
\stackrel{(41)}{\leq}-L q \delta(1-r \circ \tau)^{\gamma-1}+\delta^{\Delta} \frac{W^{\sigma}}{\delta^{\sigma}} \\
-\frac{(\gamma-1) \delta \tau^{\Delta}}{(a \circ \tau)^{1 /(\gamma-1)}\left(\delta^{\sigma}\right)^{\gamma /(\gamma-1)}}\left(W^{\sigma}\right)^{\gamma /(\gamma-1)}
\end{gathered}
$$

and then we obtain

$$
\begin{aligned}
W^{\Delta}(t) \leq & -L q(t) \delta(t)(1-r(\tau(t)))^{\gamma-1} \\
& +\frac{\delta^{\Delta}(t)}{\delta(\sigma(t))} W(\sigma(t)) \\
& -\frac{(\gamma-1) \delta(t) \tau^{\Delta}(t)}{(a(\tau(t)))^{\lambda-1}(\delta(\sigma(t)))^{\lambda}}(W(\sigma(t)))^{\lambda}
\end{aligned}
$$

on $\left[T_{0}, \infty\right)_{\mathbb{T}}$, where $\lambda=\gamma /(\gamma-1)$. Thus, for every $t, T \in$ $\left[T_{0}, \infty\right)_{\mathbb{T}}$ with $t \geq T \geq T_{0}$, by (13), we get

$$
\begin{aligned}
& \int_{T}^{t} L H(t, s) \delta(s) q(s)(1-r(\tau(s)))^{\gamma-1} \Delta s \\
& \leq H(t, T) W(T)-\int_{T}^{t}\left(-H^{\Delta_{s}}(t, s)\right) W(\sigma(s)) \Delta s \\
&+\int_{T}^{t} H(t, s) \frac{\delta^{\Delta}(s)}{\delta(\sigma(s))} W(\sigma(s)) \Delta s \\
&-\int_{T}^{t} H(t, s) \frac{(\gamma-1) \delta(s) \tau^{\Delta}(s)}{(a(\tau(s)))^{\lambda-1}(\delta(\sigma(s)))^{\lambda}}(W(\sigma(s)))^{\lambda} \Delta s
\end{aligned}
$$

$\stackrel{(28)}{=} H(t, T) W(T)$

$$
\begin{aligned}
& +\int_{T}^{t} \frac{\delta^{\Delta}(s) H(t, s)-\delta(\sigma(s)) h(t, s) H^{1 / \lambda}(t, s)}{\delta(\sigma(s))} \\
& \quad \times W(\sigma(s)) \Delta s \\
& -\int_{T}^{t} H(t, s) \frac{(\gamma-1) \delta(s) \tau^{\Delta}(s)}{(a(\tau(s)))^{\lambda-1}(\delta(\sigma(s)))^{\lambda}}(W(\sigma(s)))^{\lambda} \Delta s
\end{aligned}
$$$$
\leq H(t, T) W(T)
$$$$
+\int_{T}^{t} \frac{\delta^{\Delta}(s) H^{(\lambda-1) / \lambda}(t, s)-\delta(s) h(t, s)}{\delta(\sigma(s))}
$$$$
\times H^{1 / \lambda}(t, s) W(\sigma(s)) \Delta s
$$$$
-\int_{T}^{t} H(t, s) \frac{(\gamma-1) \delta(s) \tau^{\Delta}(s)}{(a(\tau(s)))^{\lambda-1}(\delta(\sigma(s)))^{\lambda}}(W(\sigma(s)))^{\lambda} \Delta s
$$$$
\leq H(t, T) W(T)+\int_{T}^{t} \frac{G_{+}(t, s)}{\delta(\sigma(s))} H^{1 / \lambda}(t, s) W(\sigma(s)) \Delta s
$$$$
-\int_{T}^{t} H(t, s) \frac{(\gamma-1) \delta(s) \tau^{\Delta}(s)}{(a(\tau(s)))^{\lambda-1}(\delta(\sigma(s)))^{\lambda}}(W(\sigma(s)))^{\lambda} \Delta s,
$$ 
where $G(t, s)=\delta^{\Delta}(s) H^{(\lambda-1) / \lambda}(t, s)-\delta(s) h(t, s)=\delta^{\Delta}(s) H^{1 / \gamma}$ $(t, s)-\delta(s) h(t, s), G_{+}(t, s)=\max \{0, G(t, s)\}$. So using Lemma 3, let

$$
\begin{gathered}
X=\left[H(t, s) \frac{(\gamma-1) \delta(s) \tau^{\Delta}(s)}{(a(\tau(s)))^{\lambda-1}(\delta(\sigma(s)))^{\lambda}}\right]^{1 / \lambda} W(\sigma(s)), \\
Y=\left[\frac{G_{+}(t, s)}{\lambda \delta(\sigma(s))}\left(\frac{(\gamma-1) \delta(s) \tau^{\Delta}(s)}{(a(\tau(s)))^{\lambda-1}(\delta(\sigma(s)))^{\lambda}}\right)^{-1 / \lambda}\right]^{1 /(\lambda-1)} .
\end{gathered}
$$

Using the inequality (18), we have

$$
\begin{aligned}
& \frac{G_{+}(t, s)}{\delta(\sigma(s))} H^{1 / \lambda}(t, s) W(\sigma(s)) \\
& \quad-H(t, s) \frac{(\gamma-1) \delta(s) \tau^{\Delta}(s)}{(a(\tau(s)))^{\lambda-1}(\delta(\sigma(s)))^{\lambda}}(W(\sigma(s)))^{\lambda} \\
& \quad \leq C\left(\frac{G_{+}(t, s)}{\delta(\sigma(s))}\right)^{\lambda /(\lambda-1)}\left(\frac{\delta(s) \tau^{\Delta}(s)}{(a(\tau(s)))^{\lambda-1}(\delta(\sigma(s)))^{\lambda}}\right)^{-1 /(\lambda-1)},
\end{aligned}
$$

where $C=(\lambda-1) \lambda^{-\lambda /(\lambda-1)}(\gamma-1)^{-1 /(\lambda-1)}=1 / \gamma^{\gamma}$. Thus

$$
\begin{aligned}
& \frac{G_{+}(t, s)}{\delta(\sigma(s))} H^{1 / \lambda}(t, s) W(\sigma(s)) \\
& \quad-H(t, s) \frac{(\gamma-1) \delta(s) \tau^{\Delta}(s)}{(a(\tau(s)))^{\lambda-1}(\delta(\sigma(s)))^{\lambda}}(W(\sigma(s)))^{\lambda} \\
& \quad \leq \frac{a(\tau(s))}{\gamma^{\gamma}\left(\delta(s) \tau^{\Delta}(s)\right)^{\gamma-1}} G_{+}^{\gamma}(t, s) .
\end{aligned}
$$

From (44) and (47), we obtain

$$
\begin{gathered}
\int_{T}^{t}\left[L H(t, s) \delta(s) q(s)(1-r(\tau(s)))^{\gamma-1}\right. \\
\left.\quad-\frac{a(\tau(s))}{\gamma^{\gamma}\left(\delta(s) \tau^{\Delta}(s)\right)^{\gamma-1}} G_{+}^{\gamma}(t, s)\right] \Delta s \\
\quad \leq H(t, T) W(T),
\end{gathered}
$$

that is,

$$
\begin{aligned}
\frac{1}{H(t, T)} \int_{T}^{t} & {\left[L H(t, s) \delta(s) q(s)(1-r(\tau(s)))^{\gamma-1}\right.} \\
& \left.-\frac{a(\tau(s))}{\gamma^{\gamma}\left(\delta(s) \tau^{\Delta}(s)\right)^{\gamma-1}} G_{+}^{\gamma}(t, s)\right] \Delta s \leq W(T) .
\end{aligned}
$$

From condition (32), we have

$$
\begin{gathered}
\Psi(T) \leq W(T), \quad T \in\left[T_{0}, \infty\right)_{\mathbb{T}}, \\
\limsup _{t \rightarrow \infty} \frac{1}{H(t, T)} \int_{T}^{t} L H(t, s) \delta(s) q(s)(1-r(\tau(s)))^{\gamma-1} \Delta s \\
\geq \Psi(T) .
\end{gathered}
$$

By (44), we have

$$
\begin{gathered}
\frac{1}{H(t, T)} \int_{T}^{t} L H(t, s) \delta(s) q(s)(1-r(\tau(s)))^{\gamma-1} \Delta s \\
\leq W(T)+\frac{1}{H(t, T)} \int_{T}^{t} \frac{G_{+}(t, s)}{\delta(\sigma(s))} H^{1 / \lambda}(t, s) W(\sigma(s)) \Delta s \\
-\frac{1}{H(t, T)} \int_{T}^{t} H(t, s) \frac{(\gamma-1) \delta(s) \tau^{\Delta}(s)}{(a(\tau(s)))^{\lambda-1}(\delta(\sigma(s)))^{\lambda}} \\
\times(W(\sigma(s)))^{\lambda} \Delta s .
\end{gathered}
$$

In the above inequality, take $T=T_{0}$, and write

$$
\begin{aligned}
A(t)= & \frac{1}{H\left(t, T_{0}\right)} \int_{T_{0}}^{t} \frac{G_{+}(t, s)}{\delta(\sigma(s))} H^{1 / \lambda}(t, s) W(\sigma(s)) \Delta s, \\
B(t)= & \frac{1}{H\left(t, T_{0}\right)} \\
& \times \int_{T_{0}}^{t} H(t, s) \frac{(\gamma-1) \delta(s) \tau^{\Delta}(s)}{(a(\tau(s)))^{\lambda-1}\left(\delta(\sigma(s))^{\lambda}\right.}(W(\sigma(s)))^{\lambda} \Delta s,
\end{aligned}
$$

and meanwhile noting that (50), we obtain

$$
\begin{aligned}
\liminf _{t \rightarrow \infty}[B(t)-A(t)] \\
\leq W\left(T_{0}\right)-\limsup _{t \rightarrow \infty} \frac{1}{H\left(t, T_{0}\right)} \\
\quad \times \int_{T_{0}}^{t} L H(t, s) \delta(s) q(s)(1-r(\tau(s)))^{\gamma-1} \Delta s \\
\leq W\left(T_{0}\right)-\Psi\left(T_{0}\right)<\infty .
\end{aligned}
$$

Now we assert that

$$
\int_{T_{0}}^{\infty} \frac{\delta(s) \tau^{\Delta}(s)}{(a(\tau(s)))^{\lambda-1}(\delta(\sigma(s)))^{\lambda}}(W(\sigma(s)))^{\lambda} \Delta s<\infty
$$

holds. Suppose to the contrary that

$$
\int_{T_{0}}^{\infty} \frac{\delta(s) \tau^{\Delta}(s)}{(a(\tau(s)))^{\lambda-1}(\delta(\sigma(s)))^{\lambda}}(W(\sigma(s)))^{\lambda} \Delta s=\infty,
$$

by (28), there exists a constant $\varepsilon>0$ such that

$$
\inf _{s \geq T_{0}}\left[\liminf _{t \rightarrow \infty} \frac{H(t, s)}{H\left(t, T_{0}\right)}\right]>\varepsilon>0 .
$$


From (55), there exists a $T \in\left[T_{0}, \infty\right)_{\mathbb{T}}$ for arbitrary real number $M>0$ such that

$$
\int_{T_{0}}^{t} \frac{\delta(s) \tau^{\Delta}(s)}{(a(\tau(s)))^{\lambda-1}(\delta(\sigma(s)))^{\lambda}}(W(\sigma(s)))^{\lambda} \Delta s \geq \frac{M}{(\gamma-1) \varepsilon},
$$

for $t \in[T, \infty)_{\mathbb{T}}$. By $(13)$, we have

$$
\begin{aligned}
& B(t)=\frac{1}{H\left(t, T_{0}\right)} \\
& \times \int_{T_{0}}^{t}\{(\gamma-1) H(t, s) \\
& \times\left(\int_{T_{0}}^{s} \frac{\delta(u) \tau^{\Delta}(u)}{(a(\tau(u)))^{\lambda-1}(\delta(\sigma(u)))^{\lambda}}\right. \\
& \left.\left.\times(W(\sigma(u)))^{\lambda} \Delta u\right)^{\Delta_{s}}\right\} \Delta s \\
& =\frac{1}{H\left(t, T_{0}\right)} \\
& \times \int_{T_{0}}^{t}\left\{\left[-(\gamma-1) H^{\Delta_{s}}(t, s)\right]\right. \\
& \times \int_{T_{0}}^{\sigma(s)} \frac{\delta(u) \tau^{\Delta}(u)}{(a(\tau(u)))^{\lambda-1}(\delta(\sigma(u)))^{\lambda}} \\
& \left.\times(W(\sigma(u)))^{\lambda} \Delta u\right\} \Delta s \\
& \geq \frac{1}{H\left(t, T_{0}\right)} \\
& \times \int_{T}^{t}\left\{\left[-(\gamma-1) H^{\Delta_{s}}(t, s)\right]\right. \\
& \times \int_{T_{0}}^{s} \frac{\delta(u) \tau^{\Delta}(u)}{(a(\tau(u)))^{\lambda-1}(\delta(\sigma(u)))^{\lambda}} \\
& \left.\times(W(\sigma(u)))^{\lambda} \Delta u\right\} \Delta s \\
& \geq \frac{1}{H\left(t, T_{0}\right)} \int_{T}^{t}\left[-(\gamma-1) H^{\Delta_{s}}(t, s)\right] \frac{M}{(\gamma-1) \varepsilon} \Delta s \\
& =\frac{M}{\varepsilon} \frac{H(t, T)}{H\left(t, T_{0}\right)} \text {. }
\end{aligned}
$$

From (56), there exists a $t_{2} \in[T, \infty)_{\mathbb{T}}$ such that $H(t, T) /$ $H\left(t, T_{0}\right) \geq \varepsilon$ for $t \in\left[t_{2}, \infty\right)_{\mathbb{T}}$. So $B(t) \geq M$. Since $M$ is arbitrary, we have

$$
\lim _{t \rightarrow \infty} B(t)=\infty
$$

Selecting a sequence $\left\{T_{n}\right\}_{n=1}^{\infty}: T_{n} \in\left[T_{0}, \infty\right)_{\mathbb{V}}$ with $\lim _{n \rightarrow \infty} T_{n}=\infty$ satisfying

$$
\lim _{n \rightarrow \infty}\left[B\left(T_{n}\right)-A\left(T_{n}\right)\right]=\liminf _{t \rightarrow \infty}[B(t)-A(t)]<\infty,
$$

and then there exists a constant $M_{0}>0$ such that

$$
B\left(T_{n}\right)-A\left(T_{n}\right) \leq M_{0}
$$

for sufficiently large positive integer $n$. From (59), we can easily see

$$
\lim _{n \rightarrow \infty} B\left(T_{n}\right)=\infty,
$$

and (61) implies that

$$
\lim _{n \rightarrow \infty} A\left(T_{n}\right)=\infty .
$$

From (61) and (62), we have

$$
\frac{A\left(T_{n}\right)}{B\left(T_{n}\right)}-1 \geq-\frac{M_{0}}{B\left(T_{n}\right)}>-\frac{M_{0}}{2 M_{0}}=-\frac{1}{2},
$$

that is,

$$
\frac{A\left(T_{n}\right)}{B\left(T_{n}\right)}>\frac{1}{2}
$$

for sufficiently large positive integer $n$, which together with (63) implies

$$
\lim _{n \rightarrow \infty} \frac{\left[A\left(T_{n}\right)\right]^{\gamma}}{\left[B\left(T_{n}\right)\right]^{\gamma-1}}=\lim _{n \rightarrow \infty}\left[\frac{A\left(T_{n}\right)}{B\left(T_{n}\right)}\right]^{\gamma-1} A\left(T_{n}\right)=\infty .
$$

On the other hand, by Lemma 4 , we obtain

$$
\begin{aligned}
& A\left(T_{n}\right) \frac{1}{H\left(T_{n}, T_{0}\right)} \int_{T_{0}}^{T_{n}} \frac{G_{+}\left(T_{n}, s\right)}{\delta(\sigma(s))} H^{1 / \lambda}\left(T_{n}, s\right) W(\sigma(s)) \Delta s \\
&=\int_{T_{0}}^{T_{n}}\left\{\left[\frac{(\gamma-1) H\left(T_{n}, s\right) \delta(s) \tau^{\Delta}(s)}{H\left(T_{n}, T_{0}\right)}\right]^{(\gamma-1) / \gamma}\right. \\
&\left.\quad \times \frac{W(\sigma(s))}{[a(\tau(s))]^{1 / \gamma} \delta(\sigma(s))}\right\} \\
& \times\left\{\frac{[a(\tau(s))]^{1 / \gamma} G_{+}\left(T_{n}, s\right)}{H\left(T_{n}, T_{0}\right)} H^{(\gamma-1) / \gamma}\left(T_{n}, s\right)\right. \\
&\left.\quad \times\left[\frac{(\gamma-1) H\left(T_{n}, s\right) \delta(s) \tau^{\Delta}(s)}{H\left(T_{n}, T_{0}\right)}\right]^{(1-\gamma) / \gamma}\right\} \Delta s \\
& \leq\left\{\int_{T_{0}}^{T_{n}} \frac{(\gamma-1) H\left(T_{n}, s\right) \delta(s) \tau^{\Delta}(s)}{H\left(T_{n}, T_{0}\right)}\right. \\
&\left.\times\left[\frac{W(\sigma(s))}{(a(\tau(s)))^{1 / \gamma} \delta(\sigma(s))}\right]^{\gamma /(\gamma-1)} \Delta s\right\}
\end{aligned}
$$




$$
\begin{gathered}
\times\left\{\int_{T_{0}}^{T_{n}} \frac{a(\tau(s)) G_{+}^{\gamma}\left(T_{n}, s\right)}{H^{\gamma}\left(T_{n}, T_{0}\right)} H^{\gamma-1}\left(T_{n}, s\right)\right. \\
\left.\times\left[\frac{(\gamma-1) H\left(T_{n}, s\right) \delta(s) \tau^{\Delta}(s)}{H\left(T_{n}, T_{0}\right)}\right]^{1-\gamma} \Delta s\right\}^{1 / \gamma} \\
=\left[B\left(T_{n}\right)\right]^{(\gamma-1) / \gamma}\left\{\frac{(\gamma-1)^{1-\gamma}}{H\left(T_{n}, T_{0}\right)}\right. \\
\times \int_{T_{0}}^{T_{n}} a(\tau(s)) G_{+}^{\gamma}\left(T_{n}, s\right) \\
\left.\times\left[\delta(s) \tau^{\Delta}(s)\right]^{1-\gamma} \Delta s\right\}^{1 / \gamma} .
\end{gathered}
$$

The above inequality shows that

$$
\frac{\left[A\left(T_{n}\right)\right]^{\gamma}}{\left[B\left(T_{n}\right)\right]^{\gamma-1}} \leq \frac{(\gamma-1)^{1-\gamma}}{H\left(T_{n}, T_{0}\right)} \int_{T_{0}}^{T_{n}} \frac{a(\tau(s))}{\left(\delta(s) \tau^{\Delta}(s)\right)^{\gamma-1}} G_{+}^{\gamma}\left(T_{n}, s\right) \Delta s .
$$

Hence, (66) implies

$$
\lim _{n \rightarrow \infty} \frac{1}{H\left(T_{n}, T_{0}\right)} \int_{T_{0}}^{T_{n}} \frac{a(\tau(s))}{\left(\delta(s) \tau^{\Delta}(s)\right)^{\gamma-1}} G_{+}^{\gamma}\left(T_{n}, s\right) \Delta s=\infty,
$$

which contradicts (30). Therefore (54) holds. Noting $\Psi(T) \leq$ $W(T)$ for $T \in\left[T_{0}, \infty\right)_{\mathbb{T}}$, by using (54), we obtain

$$
\begin{aligned}
& \int_{T_{0}}^{\infty} \frac{\delta(s) \tau^{\Delta}(s)}{(a(\tau(s)))^{\lambda-1}(\delta(\sigma(s)))^{\lambda}}\left(\Psi_{+}(\sigma(s))\right)^{\lambda} \Delta s \\
& \quad \leq \int_{T_{0}}^{\infty} \frac{\delta(s) \tau^{\Delta}(s)}{(a(\tau(s)))^{\lambda-1}(\delta(\sigma(s)))^{\lambda}}(W(\sigma(s)))^{\lambda} \Delta s<\infty,
\end{aligned}
$$

which is contradicting with (31). This completes the proof.

Remark 7. From Theorem 6, we can obtain different conditions for oscillation of all solutions of (1) with different choices of $\delta(t)$ and $H(t, s)$. For example, $H(t, s)=(t-s)^{m}$ or $H(t, s)=(\ln ((t+1) /(s+1)))^{m}$.
Theorem 8. Assume that $\left(H_{1}\right)-\left(H_{4}\right),(4),(28)-(29)$, and (31) hold. Suppose that $H, h, \delta$, and $\Psi$ are defined in Theorem 6. Assume that

$$
\begin{aligned}
& \liminf _{t \rightarrow \infty} \frac{1}{H\left(t, T_{0}\right)} \int_{T_{0}}^{t} L H(t, s) \delta(s) q(s)(1-r(\tau(s)))^{\gamma-1} \Delta s \\
& <\infty \\
& \liminf _{t \rightarrow \infty} \frac{1}{H(t, T)} \\
& \quad \times \int_{T}^{t}\left[L H(t, s) \delta(s) q(s)(1-r(\tau(s)))^{\gamma-1}\right. \\
& \left.\geq \Psi(T), \quad-\frac{a(\tau(s))}{\gamma^{\gamma}\left(\delta(s) \tau^{\Delta}(s)\right)^{\gamma-1}} G_{+}^{\gamma}(t, s)\right] \Delta s
\end{aligned}
$$

where $T \in\left[T_{0}, \infty\right)_{\mathbb{T}}, G(t, s)=\delta^{\Delta}(s)(H(t, s))^{1 / \gamma}-\delta(s) h(t, s)$, $G_{+}(t, s)=\max \{0, G(t, s)\}$. Then $(1)$ is oscillatory on $\left[t_{0}, \infty\right)_{\mathbb{T}}$.

Proof. Assume that (1) has a nonoscillatory solution $x(t)$ on $\left[t_{0}, \infty\right)_{\mathbb{T}}$. Without loss of generality we may assume that there exists a $t_{1} \in\left[t_{0}, \infty\right)_{\mathbb{T}}$, such that $x(t)>0$ and $x[\tau(t)]>0$ for all $t \in\left[t_{1}, \infty\right)_{\mathbb{T}}$. So $z(t)>0$ and there exists a $T_{0} \in\left[t_{1}, \infty\right)_{\mathbb{T}}$ such that

$$
x(\tau(t)) \geq(1-r(\tau(t))) z(\tau(t))
$$

for $t \in\left[T_{0}, \infty\right)_{\mathbb{T}}$. Define the function $W$ by

$$
W=\delta \frac{a\left(z^{\Delta}\right)^{\gamma-1}}{(z \circ \tau)^{\gamma-1}}, \quad t \in\left[T_{0}, \infty\right)_{\mathbb{T}} .
$$

We proceed as in the proof of Theorem 6 to obtain (44) and (47), so that

$$
\begin{aligned}
& \frac{1}{H(t, T)} \\
& \quad \times \int_{T}^{t}\left[L H(t, s) \delta(s) q(s)(1-r(\tau(s)))^{\gamma-1}\right. \\
& \left.\quad-\frac{a(\tau(s))}{\gamma^{\gamma}\left(\delta(s) \tau^{\Delta}(s)\right)^{\gamma-1}} G_{+}^{\gamma}(t, s)\right] \Delta s
\end{aligned}
$$

$\leq W(T)$ 
Hence, (72) implies

$$
\begin{gathered}
\Psi(T) \leq W(T), \quad T \in\left[T_{0}, \infty\right)_{\mathbb{T}} ; \\
\liminf _{t \rightarrow \infty} \frac{1}{H(t, T)} \int_{T}^{t} L H(t, s) \delta(s) q(s)(1-r(\tau(s)))^{\gamma-1} \Delta s \\
\geq \Psi(T) ;
\end{gathered}
$$

$\Psi(T)$

$$
\begin{aligned}
& \leq \liminf _{t \rightarrow \infty} \frac{1}{H(t, T)} \\
& \quad \times \int_{T}^{t}\left[L H(t, s) \delta(s) q(s)(1-r(\tau(s)))^{\gamma-1}\right. \\
& \left.\quad-\frac{a(\tau(s))}{\gamma^{\gamma}\left(\delta(s) \tau^{\Delta}(s)\right)^{\gamma-1}} G_{+}^{\gamma}(t, s)\right] \Delta s \\
& \quad \liminf _{t \rightarrow \infty} \frac{1}{H(t, T)} \\
& \quad \int_{T}^{t} L H(t, s) \delta(s) q(s)(1-r(\tau(s)))^{\gamma-1} \Delta s \\
& \quad \liminf _{t \rightarrow \infty} \frac{1}{H(t, T)} \int_{T}^{t} \frac{a(\tau(s))}{\gamma^{\gamma}\left(\delta(s) \tau^{\Delta}(s)\right)^{\gamma-1}} G_{+}^{\gamma}(t, s) \Delta s .
\end{aligned}
$$

From the above inequality and (71), we have

$$
\liminf _{t \rightarrow \infty} \frac{1}{H(t, T)} \int_{T}^{t} \frac{a(\tau(s))}{\left(\delta(s) \tau^{\Delta}(s)\right)^{\gamma-1}} G_{+}^{\gamma}(t, s) \Delta s<\infty
$$

Therefore, there exists a sequence $\left\{T_{n}\right\}_{n=1}^{\infty}: T_{n} \in\left[T_{0}, \infty\right)_{\mathbb{Z}}$ with $\lim _{n \rightarrow \infty} T_{n}=\infty$ such that

$$
\lim _{n \rightarrow \infty} \frac{1}{H\left(T_{n}, T\right)} \int_{T}^{T_{n}} \frac{a(\tau(s))}{\left(\delta(s) \tau^{\Delta}(s)\right)^{\gamma-1}} G_{+}^{\gamma}(t, s) \Delta s<\infty .
$$

Definitions of $A(t)$ and $B(t)$ are as in Theorem 6. From (44), and noting (77), we have

$$
\begin{aligned}
\limsup _{t \rightarrow \infty}[B(t)-A(t)] \\
\leq W\left(T_{0}\right)-\liminf _{t \rightarrow \infty} \frac{1}{H\left(t, T_{0}\right)} \\
\quad \times \int_{T_{0}}^{t} L H(t, s) \delta(s) q(s)(1-r(\tau(s)))^{\gamma-1} \Delta s \\
\leq W\left(T_{0}\right)-\Psi\left(T_{0}\right)<\infty .
\end{aligned}
$$

For the above sequence $\left\{T_{n}\right\}_{n=1}^{\infty}$,

$$
\lim _{n \rightarrow \infty}\left[B\left(T_{n}\right)-A\left(T_{n}\right)\right] \leq \limsup _{t \rightarrow \infty}[B(t)-A(t)]<\infty .
$$

We proceed by reduction to absurdity to obtain (54). The rest of the proof is similar to that of Theorem 6 and hence is omitted. This completes the proof.
If (4) is not satisfied, that is, if the condition (5) holds, we can obtain the following result.

Theorem 9. Assume that $\left(H_{1}\right)-\left(H_{4}\right),(5)$, and (28)-(32) hold. Suppose that $H, h, \delta$, and $\Psi$ are defined in Theorem 6. Assume that

$$
\int_{t_{0}}^{\infty}\left(\frac{1}{a(t)} \int_{t_{0}}^{t} q(s)[1-r(\tau(s))]^{\gamma-1} \Delta s\right)^{1 /(\gamma-1)} \Delta t=\infty
$$

holds. Then every solution $x(t)$ of (1) is either oscillatory or converges to zero on $\left[t_{0}, \infty\right)_{\mathbb{T}}$.

Proof. As the proof of Theorem 6, assume that (1) has a nonoscillatory solution $x(t)$ on $\left[t_{0}, \infty\right)_{\mathbb{T}}$. Without loss of generality we may assume that there exists $t_{1} \in\left[t_{0}, \infty\right)_{\mathbb{T}}$, such that $x(t)>0$ and $x[\tau(t)]>0$ for all $t \in\left[t_{1}, \infty\right)_{\mathbb{T}}$. So $z(t)>0$ and there exists $t_{2} \in\left[t_{1}, \infty\right)_{\mathbb{W}}$ such that

$$
x(\tau(t)) \geq(1-r(\tau(t))) z(\tau(t))
$$

for $t \in\left[t_{2}, \infty\right)_{\mathbb{T}}$. In the proof of Lemma 5 , we find that $z^{\Delta}(t)$ is either eventually positive or eventually negative. Thus, we shall distinguish the following two cases:

$$
\begin{aligned}
& \text { (I) } z^{\Delta}(t)>0 \text { for } t \in\left[t_{2}, \infty\right)_{\mathbb{T}} \\
& \text { (II) } z^{\Delta}(t)<0 \text { for } t \in\left[t_{2}, \infty\right)_{\mathbb{T}} \text {. }
\end{aligned}
$$

Case (I). When $z^{\Delta}(t)$ is eventually positive, the proof is similar to that of the proof of Theorem 6, and we can obtain that (1) is oscillatory.

Case (II). When $z^{\Delta}(t)$ is eventually negative, $z(t)$ is decreasing and $\lim _{t \rightarrow \infty} z(t)=: b \geq 0$ exists. Therefore, there exists $T_{0} \in$ $\left[t_{2}, \infty\right)_{\mathbb{T}}$, such that

$$
z(\tau(t))>z(t)>z(\sigma(t)) \geq b \geq 0
$$

for $t \in\left[T_{0}, \infty\right)_{\mathbb{T}}$. Define the function $u(t)=a(t) \mid z^{\Delta}$ $\left.(t)\right|^{\gamma-2} z^{\Delta}(t)=-a(t)\left|z^{\Delta}(t)\right|^{\gamma-1}$. Equations (1) and (85) yield

$$
\begin{aligned}
u^{\Delta}(t) & =-q(t) f\left[(x(\tau(t)))^{\gamma-1}\right] \\
& \leq-L b^{\gamma-1} q(t)(1-r(\tau(t)))^{\gamma-1}, \quad t \in\left[T_{0}, \infty\right)_{\mathbb{T}} .
\end{aligned}
$$

The inequality (86) is the assumed inequality of [8, Theorem 6.1] (see also [27, Lemma 1]). All assumptions of [8, Theorem $6.1]$ are satisfied as well. Hence the conclusion of $[8$, Theorem 6.1] holds, that is,

$$
\begin{gathered}
u(t) \leq u\left(T_{0}\right)-L b^{\gamma-1} \int_{T_{0}}^{t} q(s)(1-r(\tau(s)))^{\gamma-1} \Delta s \\
<-L b^{\gamma-1} \int_{T_{0}}^{t} q(s)(1-r(\tau(s)))^{\gamma-1} \Delta s
\end{gathered}
$$


for all $t \in\left[T_{0}, \infty\right)_{\mathbb{T}}$, and thus

$$
\begin{aligned}
\int_{T_{0}}^{l} z^{\Delta}(t) \Delta t & \\
< & -b L^{1 /(\gamma-1)} \\
& \times \int_{T_{0}}^{l}\left(\frac{1}{a(t)} \int_{T_{0}}^{t} q(s)(1-r(\tau(s)))^{\gamma-1} \Delta s\right)^{1 /(\gamma-1)} \Delta t
\end{aligned}
$$

for all $l \in\left[T_{0}, \infty\right)_{\mathbb{T}}$. Assuming $b>0$ and using (83) in (88), we can get $\lim _{l \rightarrow \infty} z(l)=-\infty$, and this is a contradiction to the fact that $z(t)>0$ for $t \in\left[t_{1}, \infty\right)_{\mathbb{T}}$. Thus $b=0$, that is $\lim _{t \rightarrow \infty} z(t)=0$. Then, it follows from $(1-r(t)) z(t) \leq$ $x(t) \leq z(t)$ that $\lim _{t \rightarrow \infty} x(t)=0$ holds. This completes the proof.

Using the same method as in the proofs of Theorems 8 and 9 , we can easily obtain the following result.

Theorem 10. Assume that $\left(H_{1}\right)-\left(H_{3}\right),(5),(28)-(29)$, (31), (71)-(72), and (83) hold. Suppose that $H, h, \delta$, and $\Psi$ are defined in Theorem 8. Then every solution $x(t)$ of $(1)$ is either oscillatory or converges to zero on $\left[t_{0}, \infty\right)_{\mathbb{T}}$.

Remark 11. The theorems in this paper are new even for the cases of $\mathbb{T}=\mathbb{R}$ and $\mathbb{T}=\mathbb{Z}$.

Example. Consider a second-order half-linear delay 2-difference equation

$$
\begin{array}{r}
{\left[\frac{1}{t^{2}}\left|z^{\Delta}(t)\right| z^{\Delta}(t)\right]^{\Delta}+\frac{1}{t^{3}}\left|x\left(\frac{t}{2}\right)\right| x\left(\frac{t}{2}\right)=0,} \\
t \in \overline{2^{\mathbb{Z}}}, \quad t \geq t_{0}:=2,
\end{array}
$$

where $z(t)=x(t)+(1 / 2) x(t / 2)$. Here, we have

$$
\begin{array}{ccc}
a(t)=\frac{1}{t^{2}}, & r(t)=\frac{1}{2}, & q(t)=\frac{1}{t^{3}}, \\
f(u)=u, & \tau(t)=\frac{t}{2}, & \gamma=3 .
\end{array}
$$

Then $\mathbb{T}=\overline{2^{\mathbb{Z}}}$ is unbounded above, $\sigma(t)=2 t$, and $\mu(t)=$ t. Conditions $\left(\mathrm{H}_{1}\right)-\left(\mathrm{H}_{3}\right)$ are clearly satisfied, and $\left(\mathrm{H}_{4}\right)$ holds with $L=1$. Next, we have

$$
\int_{2}^{t}\left[\frac{1}{a(s)}\right]^{1 /(\gamma-1)} \Delta s=\int_{2}^{t}\left[s^{2}\right]^{1 / 2} \Delta s \longrightarrow \infty \quad \text { as } t \longrightarrow \infty .
$$

Hence (4) is satisfied. Now let $H(t, s)=(t-s)^{2}$, then

$$
\begin{aligned}
H^{\Delta_{s}}(t, s) & =\frac{(t-2 s)^{2}-(t-s)^{2}}{s}=\frac{(2 t-3 s) \cdot(-s)}{s} \\
& =-(2 t-3 s)<0 \quad \forall t>s \geq t_{0}:=2 .
\end{aligned}
$$

Since

$$
\begin{aligned}
-H^{\Delta_{s}}(t, s) & =2 t-3 s=\frac{2 t-3 s}{(t-s)^{4 / 3}}\left[(t-s)^{2}\right]^{2 / 3} \\
& =\frac{2 t-3 s}{(t-s)^{4 / 3}}[H(t, s)]^{(\gamma-1) / \gamma}
\end{aligned}
$$

let $h(t, s)=(2 t-3 s) /(t-s)^{4 / 3}$; then condition (28) holds. We have

$$
\begin{aligned}
0 & <\inf _{s \geq T_{0}}\left[\liminf _{t \rightarrow \infty} \frac{H(t, s)}{H\left(t, T_{0}\right)}\right]=\inf _{s \geq T_{0}}\left[\liminf _{t \rightarrow \infty} \frac{(t-s)^{2}}{\left(t-T_{0}\right)^{2}}\right] \\
& =1<\infty \quad \forall T_{0} \in\left[t_{0}, \infty\right)_{\mathbb{T}}
\end{aligned}
$$

so condition (28) holds. Let $\delta(t)=t$ as $t \geq 2$, then $\delta^{\Delta}(t)=1$ for all $t \in\left[t_{0}, \infty\right)_{\mathbb{T}}$, and

$$
\begin{aligned}
G(t, s) & =\delta^{\Delta}(s)(H(t, s))^{1 / \gamma}-\delta(s) h(t, s) \\
& =H^{1 / 3}(t, s)-\frac{s(2 t-3 s)}{H^{2 / 3}(t, s)}<H^{1 / 3}(t, s),
\end{aligned}
$$

for all $t>s \geq 2$. Hence

$$
\begin{aligned}
& \int_{T_{0}}^{t} \frac{a(\tau(s))}{\left(\delta(s) \tau^{\Delta}(s)\right)^{\gamma-1}} G_{+}^{\gamma}(t, s) \Delta s \\
& \quad<\int_{T_{0}}^{t} \frac{(s / 2)^{-2}}{(s \cdot(1 / 2))^{2}}\left(H^{1 / 3}(t, s)\right)^{3} \Delta s=16 \int_{T_{0}}^{t} \frac{(t-s)^{2}}{s^{4}} \Delta s \\
& \quad=16\left[-\frac{8}{7 t}+\frac{8}{3 t}-\frac{2}{t}\right]-16\left[-\frac{8 t^{2}}{7 T_{0}^{3}}+\frac{8 t}{3 T_{0}^{2}}-\frac{2}{T_{0}}\right] .
\end{aligned}
$$

We get

$$
\begin{gathered}
\limsup _{t \rightarrow \infty} \frac{1}{H\left(t, T_{0}\right)} \int_{T_{0}}^{t} \frac{a(\tau(s))}{\left(\delta(s) \tau^{\Delta}(s)\right)^{\gamma-1}} G_{+}^{\gamma}(t, s) \Delta s \\
\leq \limsup _{t \rightarrow \infty}\left(16\left[-\frac{8}{7 t}+\frac{8}{3 t}-\frac{2}{t}\right]\right. \\
\left.-16\left[-\frac{8 t^{2}}{7 T_{0}^{3}}+\frac{8 t}{3 T_{0}^{2}}-\frac{2}{T_{0}}\right]\right) \\
\times\left(\left(t-T_{0}\right)^{2}\right)^{-1}=\frac{128}{7} \frac{1}{T_{0}^{3}}<\infty,
\end{gathered}
$$

and thus condition (30) holds. Let $\Psi(t)=1 / 4 t$, then

$$
\begin{aligned}
\int_{T_{0}}^{\infty} & \frac{\delta(s) \tau^{\Delta}(s)}{(a(\tau(s)))^{1 /(\gamma-1)}}\left(\frac{\Psi_{+}(\sigma(s))}{\delta(\sigma(s))}\right)^{\gamma /(\gamma-1)} \Delta s \\
\quad= & \int_{T_{0}}^{\infty} \frac{s \cdot(1 / 2)}{\left((s / 2)^{-2}\right)^{1 / 2}}\left(\frac{1 / 8 s}{2 s}\right)^{3 / 2} \Delta s=\int_{T_{0}}^{\infty} \frac{s^{2}}{4}\left(\frac{1}{16 s^{2}}\right)^{3 / 2} \Delta s \\
= & \frac{1}{256} \int_{T_{0}}^{\infty} \frac{1}{s} \Delta s=\left.\frac{1}{256} \frac{\ln s}{\ln 2}\right|_{T_{0}} ^{\infty}=\infty,
\end{aligned}
$$


that is, condition (31) holds. Since

$$
\begin{array}{rl}
\int_{T}^{t} & L H(t, s) \delta(s) q(s)(1-r(\tau(s)))^{\gamma-1} \Delta s \\
& =\frac{1}{4} \int_{T}^{t} \frac{(t-s)^{2} \cdot s}{s^{3}} \Delta s=\frac{1}{4} \int_{T}^{t}\left(\frac{t^{2}}{s^{2}}-\frac{2 t}{s}+1\right) \Delta s \\
& =\frac{1}{4}\left[-\frac{2 t^{2}}{s}-\frac{2 t \ln s}{\ln 2}+s\right]_{T}^{t} \\
& =\frac{1}{4}\left[-2 t-\frac{2 t \ln t}{\ln 2}+t\right]-\frac{1}{4}\left[-\frac{2 t^{2}}{T}-\frac{2 t \ln T}{\ln 2}+T\right],
\end{array}
$$

then

$$
\begin{aligned}
& \limsup _{t \rightarrow \infty} \frac{1}{H(t, T)} \int_{T}^{t} L H(t, s) \delta(s) q(s)(1-r(\tau(s)))^{\gamma-1} \Delta s \\
& \quad=\frac{1}{2 T} .
\end{aligned}
$$

Moreover, (97) implies

$$
\limsup _{t \rightarrow \infty} \frac{1}{H(t, T)} \int_{T}^{t} \frac{a(\tau(s))}{\gamma^{\gamma}\left(\delta(s) \tau^{\Delta}(s)\right)^{\gamma-1}} G_{+}^{\gamma}(t, s) \Delta s \leq \frac{128}{63} \frac{1}{T^{3}}
$$

Thus, when $T$ is enough large, we have

$$
\begin{aligned}
& \limsup _{t \rightarrow \infty} \frac{1}{H(t, T)} \\
& \quad \times \int_{T}^{t}\left[L H(t, s) \delta(s) q(s)(1-r(\tau(s)))^{\gamma-1}\right. \\
&\left.\quad-\frac{a(\tau(s))}{\gamma^{\gamma}\left(\delta(s) \tau^{\Delta}(s)\right)^{\gamma-1}} G_{+}^{\gamma}(t, s)\right] \Delta s \\
& \geq \frac{1}{2 T}-\frac{128}{63} \frac{1}{T^{3}} \geq \frac{1}{4 T}=\Psi(T) ;
\end{aligned}
$$

so (32) is satisfied. By Theorem 6, (89) is oscillatory on $\left[t_{0}, \infty\right)_{\mathbb{T}}$. Similarly, conditions (71) and (72) are satisfied as well. By Theorem 8, we can also obtain that (89) is oscillatory. But the other known results cannot be applied in (89).

\section{Conflict of Interests}

The authors declare that there is no conflict of interests regarding the publication of this paper.

\section{Acknowledgments}

The authors are grateful to the reviewers for their comments and suggestions. This work was supported by the Natural Science Foundation of Shandong Province of China under
Grant no. ZR2013AM003, the Development Program in Science and Technology of Shandong Province of China under Grant no. 2010GWZ20401, and the Science Foundation of Binzhou University under Grant no. BZXYQNLG201009.

\section{References}

[1] S. H. Saker, R. P. Agarwal, and D. O'Regan, "Oscillation of second-order damped dynamic equations on time scales," Journal of Mathematical Analysis and Applications, vol. 330, no. 2, pp. 1317-1337, 2007.

[2] L. Erbe, T. S. Hassan, and A. Peterson, "Oscillation criteria for nonlinear damped dynamic equations on time scales," Applied Mathematics and Computation, vol. 203, no. 1, pp. 343-357, 2008.

[3] Z. Han, W. Chen, S. Sun, and T. Li, "Oscillation behavior of a class of second-order dynamic equations with damping on time scales," Discrete Dynamics in Nature and Society, vol. 2010, Article ID 907130, 15 pages, 2010.

[4] Q. Zhang, "Oscillation of second-order half-linear delay dynamic equations with damping on time scales," Journal of Computational and Applied Mathematics, vol. 235, no. 5, pp.1180-1188, 2011.

[5] Q. Zhang and F. Qiu, "Oscillation theorems for second-order half-linear delay dynamic equations with damping on time scales," Computers \& Mathematics with Applications, vol. 62, no. 11, pp. 4185-4193, 2011.

[6] Y. B. Sun, Z. L. Han, S. R. Sun, and C. Zhang, "Oscillation of a class of second-order half-linear neutral delay dynamic equations with damping on time scales," Acta Mathematicae Applicatae Sinica, vol. 36, no. 3, pp. 480-494, 2013 (Chinese).

[7] Q. Zhang and L. Gao, "Oscillation of second-order nonlinear delay dynamic equations with damping on time scales," Journal of Applied Mathematics and Computing, vol. 37, no. 1-2, pp. 145158, 2011.

[8] M. Bohner and A. Peterson, Dynamic Equations on Time Scales: An Introduction with Applications, Birkhäuser, Boston, Mass, USA, 2001.

[9] M. Bohner and A. Peterson, Advances in Dynamic Equations on Time Scales, Birkhäuser, Boston, Mass, USA, 2003.

[10] L. Erbe, "Oscillation criteria for second order linear equations on a time scale," The Canadian Applied Mathematics Quarterly, vol. 9, no. 4, pp. 345-375, 2001.

[11] R. Agarwal, M. Bohner, D. O’Regan, and A. Peterson, "Dynamic equations on time scales: a survey," Journal of Computational and Applied Mathematics, vol. 141, no. 1-2, pp. 1-26, 2002.

[12] L. Erbe, A. Peterson, and P. Rehák, "Comparison theorems for linear dynamic equations on time scales," Journal of Mathematical Analysis and Applications, vol. 275, no. 1, pp. 418-438, 2002.

[13] M. Bohner and S. H. Saker, "Oscillation of second order nonlinear dynamic equations on time scales," The Rocky Mountain Journal of Mathematics, vol. 34, no. 4, pp. 1239-1254, 2004.

[14] S. H. Saker, "Oscillation criteria of second-order half-linear dynamic equations on time scales," Journal of Computational and Applied Mathematics, vol. 177, no. 2, pp. 375-387, 2005.

[15] R. P. Agarwal, M. Bohner, and S. H. Saker, "Oscillation of second order delay dynamic equations," The Canadian Applied Mathematics Quarterly, vol. 13, no. 1, pp. 1-18, 2005.

[16] Y. Şahiner, "Oscillation of second-order delay differential equations on time scales," Nonlinear Analysis: Theory, Methods and Applications, vol. 63, no. 5-7, pp. e1073-e1080, 2005. 
[17] L. Erbe, A. Peterson, and S. H. Saker, "Oscillation criteria for second-order nonlinear delay dynamic equations," Journal of Mathematical Analysis and Applications, vol. 333, no. 1, pp. 505522, 2007.

[18] T. S. Hassan, "Oscillation criteria for half-linear dynamic equations on time scales," Journal of Mathematical Analysis and Applications, vol. 345, no. 1, pp. 176-185, 2008.

[19] S. Sun, Z. Han, and C. Zhang, "Oscillation of second-order delay dynamic equations on time scales," Journal of Applied Mathematics and Computing, vol. 30, no. 1-2, pp. 459-468, 2009.

[20] S. R. Grace, M. Bohner, and R. P. Agarwal, "On the oscillation of second-order half-linear dynamic equations," Journal of Difference Equations and Applications, vol. 15, no. 5, pp. 451-460, 2009.

[21] L. Erbe, T. S. Hassan, and A. Peterson, "Oscillation criteria for nonlinear functional neutral dynamic equations on time scales," Journal of Difference Equations and Applications, vol. 15, no. 1112, pp. 1097-1116, 2009.

[22] D. R. Anderson and A. Zafer, "Nonlinear oscillation of secondorder dynamic equations on time scales," Applied Mathematics Letters, vol. 22, no. 10, pp. 1591-1597, 2009.

[23] S. R. Grace, R. P. Agarwal, B. Kaymakçalan, and W. Saejie, "Oscillation theorems for second order nonlinear dynamic equations," Journal of Applied Mathematics and Computing, vol. 32, no. 1, pp. 205-218, 2010.

[24] Ch. G. Philos, "Oscillation theorems for linear differential equations of second order," Archiv der Mathematik, vol. 53, no. 5, pp. 482-492, 1989.

[25] G. H. Hardy, J. E. Littlewood, and G. Pólya, Inequalities, Cambridge University Press, Cambridge, UK, 2nd edition, 1988.

[26] A. Tuna and S. Kutukcu, "Some integral inequalities on time scales," Applied Mathematics and Mechanics, vol. 29, no. 1, pp. 23-29, 2008.

[27] M. Bohner, "Some oscillation criteria for first order delay dynamic equations," Far East Journal of Applied Mathematics, vol. 18, no. 3, pp. 289-304, 2005. 


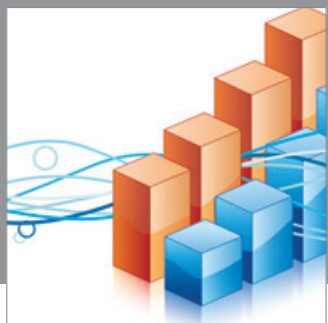

Advances in

Operations Research

mansans

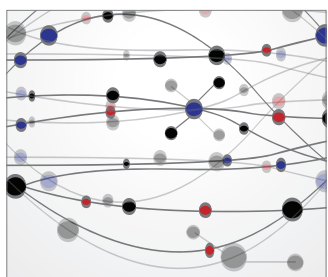

The Scientific World Journal
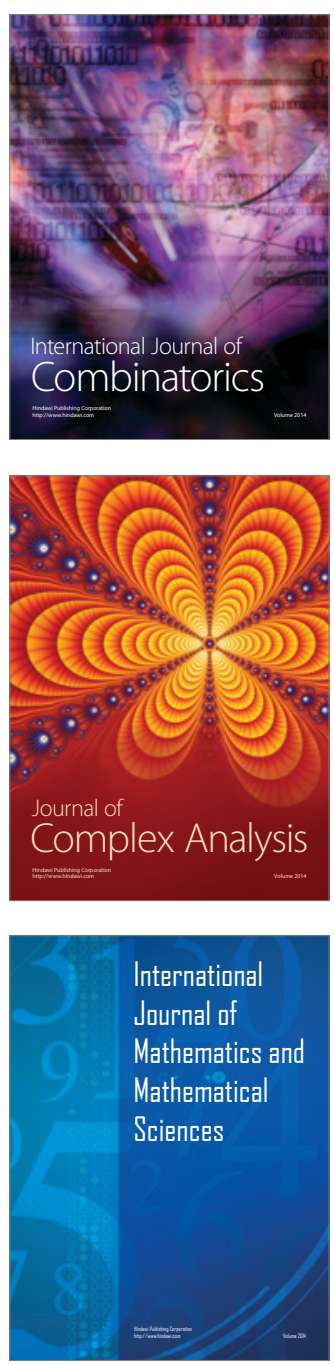
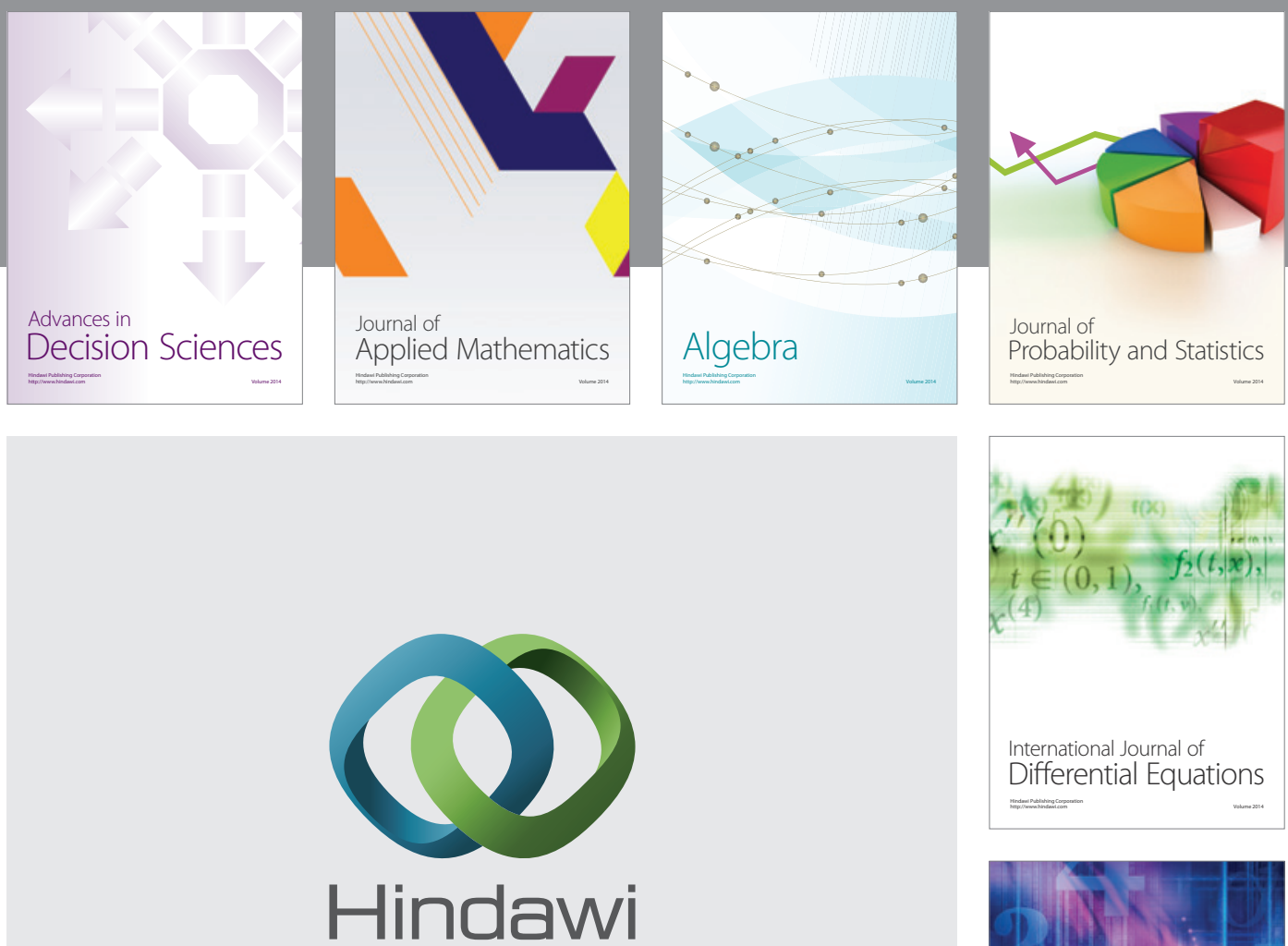

Submit your manuscripts at http://www.hindawi.com
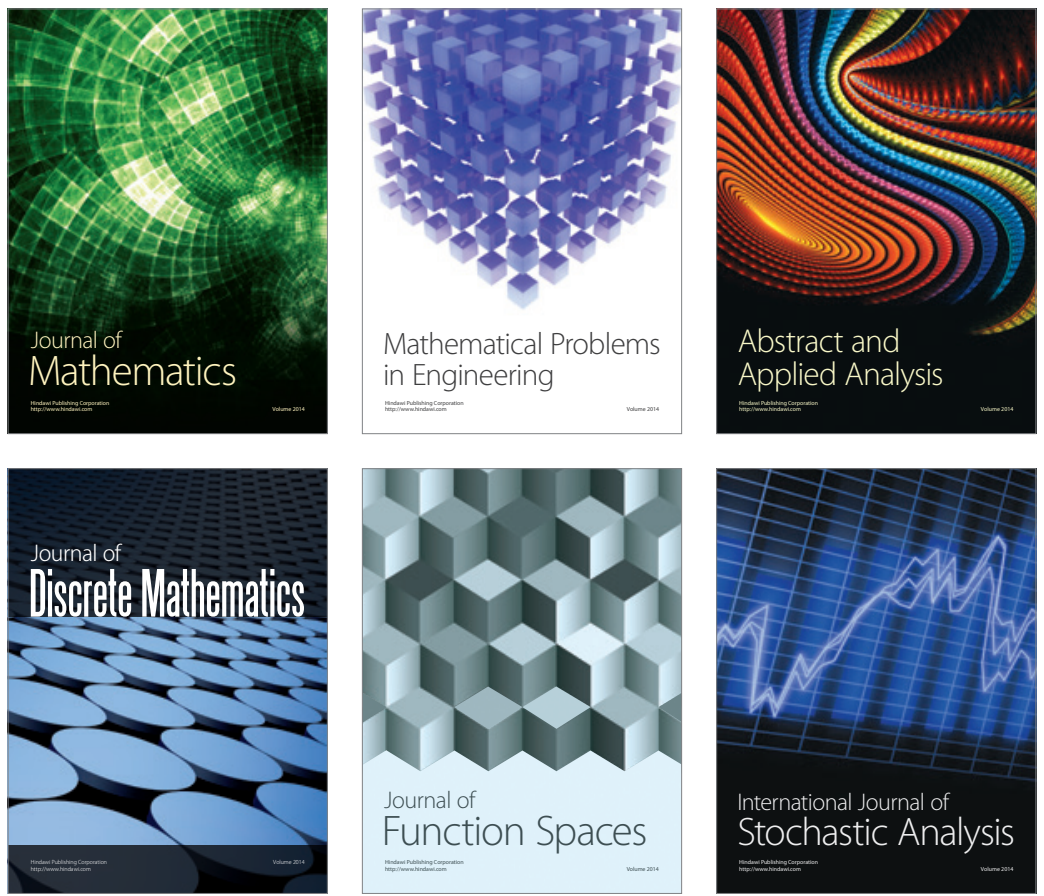

Journal of

Function Spaces

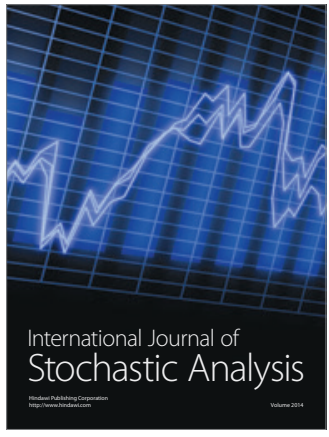

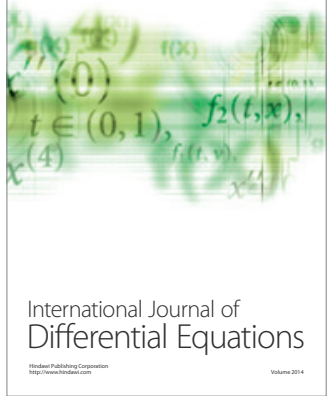
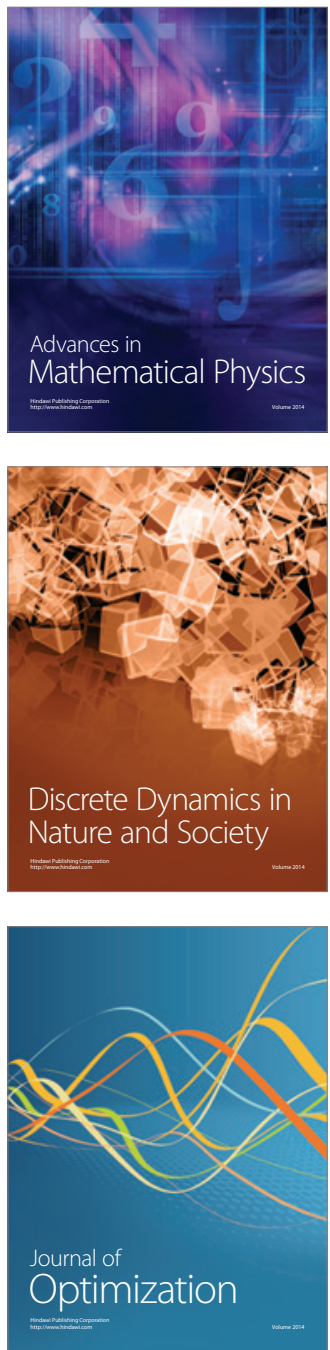